\title{
ESTUDO COMPARATIVO ENTRE O MÉTODO SIMPLEX E O MÉTODO EVOLUTIONARY PARA A OTIMIZAÇÃO DE ROTAS DE DISTRIBUIDORES NA CIDADE DE SÃO JOSÉ DO RIO PRETO - SP
}

\section{COMPARATIVE STUDY BETWEEN METHOD SIMPLEX AND METHOD EVOLUTIONARY FOR THE OPTIMIZATION OF DISTRIBUTORS ROUTES IN THE CITY OF SÃO JOSÉ DO RIO PRETO - SP}

Carolaine Milena Paulino da Silva - carolaine.milena@ outlook.com Carlos Eduardo Vechiato Neves - elys.du@hotmail.com Douglas José de Oliveira - douglasjo@outlook.com Eduardo Fraiha Neto - eduardo.fraiha@fatecbb.edu.br Oswaldo Lazaro Mendes - oswaldo.lazaro@fatecbb.edu.br Faculdade de Tecnologia de Bebedouro (FATEC) - SP - Brasil

DOI: 10.31510/infa.v15i2.489

\section{RESUMO}

Esse trabalho tem por objetivo demonstrar de uma maneira compreensível a comparação entre os métodos simplex e evolutionary para encontrar o caminho mais curto em três pontos de partidas estratégicos na cidade de São José do Rio Preto - SP. Para a elaboração do trabalho foram desenvolvidas algumas rotas procurando-se os caminhos mais curtos entre quinze distribuidoras de bebidas em diferentes pontos estratégicos da cidade utilizando como ferramenta o Solver do Excel. Durante o desenvolvimento dos roteiros utilizando o método simplex, foi observado o grau exponencial de dificuldade em montar o problema no Excel, utilizando apenas um importante ponto de partida para três distribuidoras de bebidas. Já no método evolutionary percebeu-se que podem ser utilizados mais pontos de partidas para uma quantidade maior de distribuidoras de bebidas. Conclui-se que no dia a dia de uma empresa que necessita de agilidade na distribuição, há ferramentas como os métodos utilizados para a execução de sua logística de distribuição que fornecem informações relevantes para a tomada de decisões.

Palavras-chave: Método Simplex, Método Evolutionary, Roteirização.

\begin{abstract}
This work aims to demonstrate in a clear way the comparison between simplex and evolutionary methods to find the shortest path in three strategic starting points in the city of São José do Rio Preto. To elaborate this work, some routes were developed searching for the shortest paths among fifteen distributors of drinks in different strategic points of the city using Excel Solver the main tool. During the development of the scripts using the simplex method, it was observed
\end{abstract}


the exponential degree of difficulty in setting up the problem on Excel, using only one important starting points for three beverage distributors. On the other hand, in the evolutionary method, it was noticed that more starting points can be used for a larger number of beverage distributors. It is concluded that in the routine of a company that needs agility in the distribution, there are tools like the methods used for the execution of its distribution logistics that provide relevant information to the decision making.

Keywords: Simplex method, Evolutionary method, Routing

\section{INTRODUÇÃ̃o}

Esse estudo tem o objetivo de avaliar a importância e as vantagens de utilizar métodos diferentes para se encontrar o caminho mais curto para distribuição de bebidas na cidade de São José do Rio Preto - SP. Em um mercado competitivo é necessário que as empresas busquem melhores soluções a fim de conseguir certa economia e rapidez em seus processos logísticos, trazendo benefícios para si e principalmente para seus clientes.

Para a obtenção de resultados positivos na otimização da utilização de uma frota, podese usar as ferramentas disponíveis no Microsoft Excel para a solução de problemas, no que tange a minimização dos trajetos, como a ferramenta solver, que utiliza os métodos evolutionary e simplex.

Para alcançar tal objetivo, a metodologia desse estudo se define primeiro por uma breve revisão em livros e pesquisas acadêmicas, a fim de compreender os principais métodos de roteirização com o propósito de escolher a ferramenta mais eficaz para a obtenção de melhores resultados. A seguir, no intuito de verificar na prática os conceitos levantados, apresenta-se uma análise prática de como uma empresa do setor de distribuição de bebidas pode obter os melhores resultados, utilizando, neste caso, as ferramentas adequadas nos processos de distribuição para evitar possíveis gastos desnecessários, garantindo assim uma distribuição concisa.

Para introduzir a fundamentação teórica desse artigo, as páginas a seguir estabelecem um resgate de como os processos de distribuições ganharam formas mais eficazes utilizandose recursos facilitadores.

\section{FUNDAMENTAÇÃO TEÓRICA}




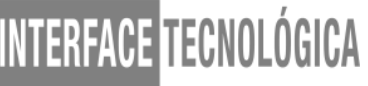

Segundo Colin (2017), existem vários softwares para a resolução de problemas de programação linear. Colin (2017) diz ainda que há dois grandes grupos, os de grande flexibilidade e os de baixa flexibilidade. O primeiro é aquele em que serve como suplementos de planilhas eletrônicas; já o segundo são os outros, que são usados em aplicações mais intensivas e dedicadas.

Colin (2017), explica que há vários suplementos como por exemplo Frontline Systems (Solver), Palisade Corporation (Evolver), LINDO Systems; e ainda existem vários softwares de alta flexibilidade, como por exemplo: AIMSS, AMPL, GAMS, MATLAB e MPL.

Para que a modelagem seja feita no solver deve-se inserir as características do problema, dando origem a função objetivo, as variáveis e as restrições. Após inseridas, o solver fornece uma saída dando a solução ótima do problema. (COLIN 2017)

Já para Lachtermacher (2009), uma das ferramentas que vem a contribuir significativamente são as planilhas eletrônicas como por exemplo o Microsoft Excel, o Lotus e o Quattro-Pró. Para resolver problemas no Solver, que é uma ferramenta do Microsoft Excel, deve-se inserir os parâmetros do problema, como as restrições e a função objetivo. Para que haja exatidão na solução do problema, é necessário que sejam inseridas as informações corretas e coerentes.

Para Andrade (2014), o avanço da pesquisa operacional se deu graças ao desenvolvimento dos computadores e também a disseminação dos microcomputadores contribuindo com a análise de decisões.

A pesquisa operacional possui um enfoque clássico, onde se busca a solução ótima, e o enfoque atual, onde se usa o modelo a fim de identificar o problema certo.

Andrade (2014) diz que existem várias fases em um problema de pesquisa operacional. São elas: definição do problema, construção do modelo, solução do modelo, validação do modelo, implementação da solução, e finalmente a avaliação final.

Segundo Caixeta-Filho (2015, p.10), “as técnicas de programação matemática, em particular a programação linear [...], enquadram-se sob a óptica de modelagem normativa".

De acordo com Moreira (2015), a pesquisa operacional serve para desenvolver problemas a fim de conduzir e coordenar determinados processos em uma organização. Para que a pesquisa operacional possa ter sucesso na resolução do problema, deve-se observar o problema e modelar o mesmo. 


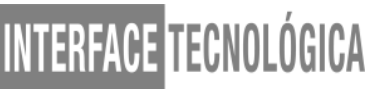

Para Colin (2017), o algoritmo simplex utiliza várias definições e conceitos relacionados, sendo importante utilizar parte desses conceitos em separado para que a compreensão do algoritmo seja melhor entendida.

Para um problema em sua forma padrão, o algoritmo simplex vai de uma solução viável para outra, de forma que o valor da função objetivo é minimizado até o ponto ótimo ser alcançado. $\mathrm{O}$ algoritmo pode ser dividido em três partes: o algoritmo prepara os dados de entrada; o algoritmo repete diversas vezes o procedimento e faz com que a otimização do modo seja completada (iteração); o algoritmo avalia se a solução ótima foi obtida, ou se é impossível obtê-la (regra de parada). (COLIN 2017)

Colin (2017, p. 21) ainda diz que "[...] a solução dos problemas de programação linear é feita por meio de algoritmos, e em especial pelo algoritmo simplex".

Para Moreira (2015), o método simplex consiste em uma sequência de cálculos que levam a resolução de um problema de programação linear. Para tanto, deve-se seguir os seguintes passos:

- Criar uma tabela inicial, onde o valor da função objetivo corresponde a zero;

- Transformar o primeiro em um segundo com uma solução melhorada;

- Repetir a forma até encontrar a solução ótima;

- Verificar se a solução ótima foi atingida.

De acordo com Andrade (2014), a ferramenta simplex é um método que pode ser utilizado para resolver problemas de separação de recursos e consiste nos seguintes processos: formulação do problema, montagem do modelo e solução do modelo.

Para Caixeta-Filho (2015) o método simplex responde questões de como a função objetivo deve ser tratada a fim de se encontrar uma solução ótima para o problema.

Segundo Lanes e Greco (2013), o método evolutionary é uma ótima alternativa para encontrar uma solução para determinado problema, sendo que para problemas estruturais o método Otimização Estrutural Evolucionária tem sido o mais utilizado. O método foi proposto por Xie e Steven (1993), baseando-se em uma aplicação do Método dos Elementos Finitos, chamado de MEF. Esta fórmula foi desenvolvida partindo de um algoritmo evolucionário baseado em uma formulação que se sustenta na colocação de elementos pouco utilizados durante o processo. Este procedimento é chamado hard-kill. Para os autores, o método evolucionário ficou famoso por ser um algoritmo simples e de fácil inserção. 


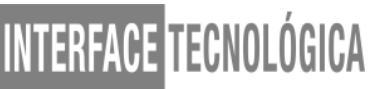

\section{PROCEDIMENTOS METODOLÓGICOS}

Para o desenvolvimento do presente artigo foram utilizadas pesquisa bibliográfica, que segundo Gil (2002) é feita por dados obtidos em fontes como artigos científicos e livros e caracteriza-se como pesquisa científica exploratória.

O seguinte estudo contempla o desenvolvimento de várias planilhas utilizando a ferramenta Solver do Microsoft Excel para encontrar o caminho mais curto em três pontos de partidas estratégicos na cidade de São José do Rio Preto e foi feito inicialmente para sete pontos, sendo uma entrada mais 6 pontos, depois para 11 pontos, sendo uma entrada mais 10 pontos e por fim 16 pontos, sendo uma entrada e mais 15 pontos utilizando o Método Evolutionary e um com apenas 4 pontos utilizando o Método Simplex, devido à complexidade para esse tipo aplicação. Para encontrar as distâncias entre os pontos foi utilizada a ferramenta Google Maps e foi feita uma comparação entre os dois métodos: O Método Simplex e o Método Evolutionary.

\section{RESULTADOS E DISCUSSÃO}

O intuito do trabalho foi descobrir caminhos mais curtos entre algumas distribuidoras de bebidas utilizando métodos que viessem a facilitar na roteirização de trajetos diferentes. As rotas foram traçadas tendo como ponto de partida, três principais entradas na cidade de São José do Rio Preto em pontos diversificados de extremo a extremo. Inicialmente partiu-se da Avenida Adalberto Andaló passando por seis distribuidoras diferentes e retornando para o ponto de partida.

Para tal foi utilizada a ferramenta Google Maps para localizar as distâncias entre os pontos, e, então, depois de montadas as distâncias foi utilizado o método evolutionary trazendo melhores resultados no que diz respeito as rotas originais, pois foram encontrados caminhos mais curtos, reduzindo, assim os custos.

Também foram utilizadas mais duas entradas diferentes, uma com dez e a outra com quinze distribuidoras diferentes. Foi observado que o método evolutionary é um facilitador independentemente da quantidade de pontos entre a partida e a chegada. No Método Simplex foi feita uma planilha com 4 pontos devido à complexidade em trabalhar com muitos pontos.

Na Figura 1 tem-se 6 distribuidoras de bebidas e ponto de partida e chegada, Entrada 1 
Figura 1-: 6 distribuidoras de bebidas e a Entrada 1

\begin{tabular}{|l|l|}
\hline & \multicolumn{1}{|c|}{ Nome dos Pontos de Entrega de Bebidas } \\
\hline 1 & Serv Festa São Paulo \\
\hline 2 & Bertasso Distribuidora de Águas Minerais \\
\hline 3 & RH de Lima Bebidas \\
\hline 4 & Vilar Comércio de Bebidas \\
\hline 5 & Manauscol Distribuidora de Bebidas \\
\hline 6 & Elizabete Oliveira Distribuidora de Bebidas \\
\hline 7 & Avenida Andaló (Entrada 1) \\
\hline & Fonte: Elaborada pelos Autores (2018)
\end{tabular}

Na Tabela 1 tem-se os cálculos das distâncias para as 6 distribuidoras e o ponto de partida e chegada, Entrada 1 (Av. Andaló).

Tabela 1 -: Cálculo das distâncias para 6 distribuidoras e Entrada 1 (Av. Andaló)

\begin{tabular}{|c|c|c|c|c|c|c|c|c|}
\hline & Bebida 1 & \begin{tabular}{|l|} 
Bebida 2 \\
\end{tabular} & \begin{tabular}{|l|} 
Bebida 3 \\
\end{tabular} & Bebida 4 & \begin{tabular}{|l|} 
Bebida 5 \\
\end{tabular} & Bebida 6 & Andaló & \\
\hline & 1 & 2 & 3 & 4 & 5 & 6 & 7 & \\
\hline 1 & & 4,11 & 3,2 & 5,9 & 7,8 & 6,1 & 3,5 & \\
\hline 2 & 4,1 & & 2,9 & 4,2 & 5,5 & 10,4 & 3,1 & \\
\hline 3 & 2,4 & 2,7 & & 4,4 & 2,3 & 3 & 1,5 & \\
\hline 4 & 5,6 & 4,3 & 4,1 & & 3,1 & 6,6 & 5,5 & \\
\hline 5 & 4,4 & 4,4 & 2,7 & 3,6 & & 2,8 & 1,5 & \\
\hline 6 & 5,5 & 5,2 & 3,4 & 8,5 & 3,3 & & 3,8 & \\
\hline 7 & 3,5 & 3,1 & 1,5 & 5,5 & 1,5 & 3,8 & & \\
\hline & & & & & & & & \\
\hline \multicolumn{9}{|c|}{ Cálculos de distâncias utilizando o google maps } \\
\hline \multirow[t]{3}{*}{7} & 1 & 2 & 3 & 4 & 5 & 6 & & Distâncias \\
\hline & 3,5 & 4,11 & 2,9 & 4,4 & 3,1 & 2,8 & 3,8 & 24,61 \\
\hline & \multicolumn{8}{|c|}{ Cálculos de distâncias utilizando o método evolutionary } \\
\hline 7 & 3 & 1 & 2 & 4 & 5 & 6 & & Distâncias \\
\hline & 1,5 & 2,4 & 4,11 & 4,2 & 3,1 & 2,8 & 3,8 & 21,91 \\
\hline
\end{tabular}

Fonte: Elaborada pelos Autores (2018)

De acordo com a Tabela 1 o melhor caminho entre as distâncias em relação a distância proposta foi de aproximadamente $11 \%$, ou seja, ocorreu um ganho de $11 \%$ no percurso.

Na Figura 2 tem-se as 10 distribuidoras de bebidas e o ponto de partida e chegada, entrada 2, (Rod Transbrasiliana - Shopping Iguatemi).

Figura 2-: 10 distribuidoras de bebidas e a Entrada 2

\begin{tabular}{|l|l|}
\hline & \multicolumn{1}{|c|}{ Nome dos Pontos de Entrega de Bebidas } \\
\hline 1 & Serv Festa São Paulo \\
\hline 2 & Bertasso Distribuidora de Águas Minerais \\
\hline
\end{tabular}


WWTEPFACETEENOLOLGCA

\begin{tabular}{|c|l|}
\hline 3 & RH de Lima Bebidas \\
\hline 4 & Vilar Comércio de Bebidas \\
\hline 5 & Manauscol Distribuidora de Bebidas \\
\hline 6 & Elizabete Oliveira Distribuidora de Bebidas \\
\hline 7 & DBK Distribuidora de Bebidas \\
\hline 8 & Lig Chopp Germânia \\
\hline 9 & Fernandão Bebidas \\
\hline 10 & Cofraria Conebel \\
\hline 11 & $\begin{array}{l}\text { Rodovia Transbrasiliana - Shopping Iguatemi } \\
\text { (Entrada 2) - Ponto de Partida e Chegada }\end{array}$ \\
\hline
\end{tabular}

Fonte: Elaborada pelos Autores (2018)

No Tabela 2 tem-se os cálculos das distâncias para 10 distribuidoras e o ponto de partida e chegada, Entrada 2.

Tabela 2-Cálculos das distâncias para 10 distribuidoras e a Entrada 2

\begin{tabular}{|c|c|c|c|c|c|c|c|c|c|c|c|c|}
\hline & Bebida 1 & Bebida 2 & Bebida 3 & Bebida 4 & Bebida 5 & Bebida 6 & Bebida 7 & Bebida 8 & Bebida 9 & Bebida 10 & Entrada 2 & \\
\hline & 1 & 2 & 3 & 4 & 5 & 6 & 7 & 8 & 9 & 10 & 11 & \\
\hline 1 & & 4,11 & 3,2 & 5,9 & 7,8 & 6,1 & 7,1 & 5,7 & 4,9 & 7,6 & 11,3 & \\
\hline 2 & 4,1 & & 2,9 & 4,2 & 5,5 & 10,4 & 10,4 & 6,1 & 5,2 & 4 & 9,8 & \\
\hline 3 & 2,4 & 2,7 & & 4,4 & 2,3 & 3 & 6,9 & 4,1 & 3 & 2,1 & 9,6 & \\
\hline 4 & 5,6 & 4,3 & 4,1 & & 3,1 & 6,6 & 10,1 & 1 & 5 & 2,8 & 6,7 & \\
\hline 5 & 4,4 & 4,4 & 2,7 & 3,6 & & 2,8 & 8,1 & 3,4 & 2 & 0,3 & 7,4 & \\
\hline 6 & 5,5 & 5,2 & 3,4 & 8,5 & 3,3 & & 6,9 & 6,2 & 0,9 & 3,2 & 9 & \\
\hline 7 & 7,1 & 10,6 & 7 & 12,1 & 8,1 & 6,5 & & 9,8 & 7 & 7,8 & 17,4 & \\
\hline 8 & 6 & 4,6 & 4,4 & 1,4 & 3,4 & 6,4 & 10,3 & & 6,3 & 3,1 & 6,4 & \\
\hline 9 & 4,7 & 4,9 & 2,9 & 8,4 & 2,5 & 0,85 & 6,8 & 6,1 & & 2,4 & 9,3 & \\
\hline 10 & 4,1 & 4,2 & 2,5 & 3 & 0,85 & 3,6 & 8,5 & 2,7 & 2,7 & & 8,4 & \\
\hline 11 & 11,3 & 9,8 & 9,6 & 6,7 & 7,4 & 9 & 17,4 & 6,4 & 9,3 & 8,4 & & \\
\hline \multicolumn{13}{|c|}{ Cálculos de distâncias utilizando o google maps } \\
\hline 11 & 1 & 2 & 3 & 4 & 5 & 6 & 7 & 8 & 9 & 10 & & \multirow{2}{*}{\begin{tabular}{|c|} 
Distâncias \\
62,41 \\
\end{tabular}} \\
\hline & 11,3 & 4,11 & 2,9 & 4,4 & 3,1 & 2,8 & 6,9 & 9,8 & 6,3 & 2,4 & 8,4 & \\
\hline \multicolumn{13}{|c|}{ Cálculos de distâncias utilizando o método evolutionary } \\
\hline 11 & 8 & 4 & 2 & 3 & 1 & 7 & 6 & 9 & 10 & 5 & & \multirow{2}{*}{$\begin{array}{c}\text { Distâncias } \\
42,55\end{array}$} \\
\hline & 6,4 & 1,4 & 4,3 & 2,9 & 2,4 & 7,1 & 6,5 & 0,9 & 2,4 & 0,85 & 7,4 & \\
\hline
\end{tabular}

Fonte: Elaborada pelos Autores (2018)

De acordo com o Tabela 2 o melhor caminho entre as distâncias em relação a distância proposta foi de aproximadamente $32 \%$, ou seja, ocorreu um ganho de $32 \%$ no percurso.

Na Figura 3 tem-se as 15 distribuidoras e o ponto de partida e chegada, Entrada 3.

Figura 3-: 15 distribuidoras de bebidas e a Entrada 3

\begin{tabular}{|l|l|}
\hline & \multicolumn{1}{|c|}{ Nome dos Pontos de Entrega de Bebidas } \\
\hline 1 & Serv Festa São Paulo \\
\hline
\end{tabular}




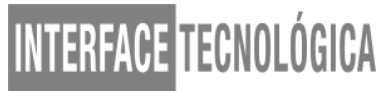

\begin{tabular}{|c|l|}
\hline 2 & Bertasso Distribuidora de Águas Minerais \\
\hline 3 & RH de Lima Bebidas \\
\hline 4 & Vilar Comércio de Bebidas \\
\hline 5 & Manauscol Distribuidora de Bebidas \\
\hline 6 & Elizabete Oliveira Distribuidora de Bebidas \\
\hline 7 & DBK Distribuidora de Bebidas \\
\hline 8 & Lig Chopp Germânia \\
\hline 9 & Fernandão Bebidas \\
\hline 10 & Cofraria Conebel \\
\hline 11 & Cofan Distribuidora de Bebidas \\
\hline 12 & Refrigerantes Arco Iris \\
\hline 13 & LB Bier Distribuidora de Bebidas \\
\hline 14 & Praiamar Indústria Comércio Distribuição \\
\hline 15 & Spal S/A Indústria Brasileira de Bebidas \\
\hline 16 & Rodovia Assis Chateaubriand - TV TEM \\
\hline
\end{tabular}

Fonte: Elaborada pelos Autores (2018)

Na Tabela 3 tem-se os cálculos das distâncias para as 15 distribuidoras e o ponto de partida e chegada, Entrada 3 (Rodovia Assis Chauteaubriand - TV TEM) 
[NTERFACEETECENOĹGGCA

Tabela 3-: Cálculos das distâncias para 15 distribuidoras e a Entrada 3.

\begin{tabular}{|c|c|c|c|c|c|c|c|c|c|c|c|c|c|c|c|c|c|}
\hline & Bebida 1 & Bebida 2 & Bebida 3 & Bebida 4 & Bebida 5 & Bebida 6 & Bebida 7 & Bebida 8 & Bebida 9 & Bebida 10 B & Bebida 11 & Bebida 12 & Bebida 13 & Bebida 14 & Bebida 15 & Entrada 3 & \\
\hline & 1 & 2 & 3 & 4 & 5 & 6 & 7 & 8 & 9 & 10 & 11 & 12 & 13 & 14 & 15 & 16 & \\
\hline 1 & & 4,11 & 3,2 & 5,9 & 7,8 & 6,1 & 7,1 & 5,7 & \begin{tabular}{l|l}
, 9 \\
\end{tabular} & \begin{tabular}{ll|}
7,6 \\
\end{tabular} & 12,3 & 6,2 & 1,7 & \begin{tabular}{l|l}
6 \\
\end{tabular} & 2,3 & 3,1 & \\
\hline 2 & 4,1 & & 2,9 & 4,2 & 5,5 & 10,4 & 10,4 & 6,1 & 5,2 & 4 & 4,9 & 3,1 & 5,5 & 2,5 & 3 & 2,7 & \\
\hline 3 & 2,4 & 2,7 & & 4,4 & 2,3 & 3 & 6,9 & 4,1 & 3 & 2,1 & 6,1 & 5,8 & 3,6 & 7 & 6 & 6,6 & \\
\hline 4 & 5,6 & 4,3 & 4,1 & & 3,1 & 6,6 & 10,1 & 1 & 5 & 2,8 & 3,5 & 3,2 & 10,4 & 4,3 & 3,3 & 4,3 & \\
\hline 5 & 4,4 & 4,4 & 2,7 & 3,6 & & 2,8 & 8,1 & 3,4 & 2 & 0,3 & 7,2 & 6,2 & 9,1 & 7,4 & 6,4 & 7,7 & \\
\hline 6 & 5,5 & 5,2 & 3,4 & 8,5 & 3,3 & & 6,9 & 6,2 & 0,9 & 3,2 & 9 & 7,9 & 7,3 & 9,1 & 8,1 & 5,9 & \\
\hline 7 & 7,1 & 10,6 & 7 & 12,1 & 8,1 & 6,5 & & 9,8 & 7 & 7,8 & 12,5 & 11,5 & 5,8 & 12,7 & 11,7 & 7,2 & \\
\hline 8 & 6 & 4,6 & 4,4 & 1,4 & 3,4 & 6,4 & 10,3 & & 6,3 & 3,1 & 3,8 & 3,4 & 10,7 & $\begin{array}{l}4,6 \\
\end{array}$ & 3,6 & 8,8 & \\
\hline 9 & 4,7 & 4,9 & 2,9 & 8,4 & 2,5 & 0,85 & 6,8 & 6,1 & & 2,4 & 8,9 & 7,8 & 7,2 & 9 & 8 & 6,5 & \\
\hline 10 & 4,1 & 4,2 & 2,5 & 3 & 0,85 & 3,6 & 8,5 & 2,7 & 2,7 & & 4,8 & 4,4 & 8,8 & 7,1 & 6,1 & 7,4 & \\
\hline 11 & 11,3 & 9,8 & 9,6 & 6,7 & 7,4 & 9 & 17,4 & 6,4 & 9,3 & 8,4 & & 1,9 & 11,2 & 2,2 & 2 & 12,2 & \\
\hline 12 & 11,3 & 3,1 & 5,8 & 3,2 & 6,2 & 7,9 & 11,5 & 3,4 & 7,8 & 4,4 & 1,1 & & 10,2 & 1,1 & 0,3 & 11,1 & \\
\hline 13 & 1,7 & 5,4 & 3,6 & 10,4 & 9,1 & 7,3 & 5,8 & 10,7 & 7,2 & 8,8 & 12,4 & 12,5 & & 13,4 & 12,6 & 2,1 & \\
\hline 14 & 6 & 2,5 & 7 & 4,3 & 7,4 & 9,1 & 12,7 & 4,6 & 9 & 5,6 & 2,1 & 1 & 11,3 & & 0,8 & 12,3 & \\
\hline 15 & 6,1 & 3 & 6 & 3,3 & 6,4 & 8,1 & 11,7 & 3,6 & 8,9 & 4,6 & 1,3 & 0,3 & 10,3 & 0,8 & & 11,3 & \\
\hline 16 & 3,1 & 2,7 & 6,6 & 4,3 & 7,7 & 5,9 & 7,2 & 8,8 & 6,5 & 7,4 & 12,2 & 11,1 & 2,1 & 12,3 & 11,3 & & \\
\hline \multirow{2}{*}{\multicolumn{18}{|c|}{ Cálculos de distâncias utilizando o google maps }} \\
\hline & & & & & & & & & & & & & & & & & \\
\hline 16 & 1 & 2 & 3 & 4 & 5 & 6 & 7 & 8 & 9 & 10 & 11 & 12 & 13 & 14 & 15 & & Distâncias \\
\hline & 3,1 & 4,11 & 2,9 & 4,4 & 3,1 & 2,8 & 6,9 & 9,8 & 6,3 & 2,4 & 4,8 & 1,9 & 10,2 & 13,4 & 0,8 & 11,3 & 88,21 \\
\hline \multirow{2}{*}{\multicolumn{18}{|c|}{ Cálculos de distâncias utilizando o método evolutionary }} \\
\hline & & & & & & & & & & & & & & & & & \\
\hline 16 & 13 & 7 & 6 & 9 & 5 & 10 & 8 & 4 & 11 & 12 & 15 & 14 & 2 & 3 & 1 & t & Distâncias \\
\hline & 2,1 & 5,8 & 6,5 & 0,9 & 2,5 & 0,3 & 2,7 & 1,4 & 3,5 & 1,9 & 0,3 & 0,8 & 2,5 & 2,9 & 2,4 & 3,1 & 39,6 \\
\hline
\end{tabular}

Fonte: Elaborada pelos Autores (2018)

De acordo com o Tabela 3 o melhor caminho entre as distâncias em relação a distância proposta foi de aproximadamente 55\%, ou seja, ocorreu um ganho de 55\% no percurso, sendo assim percorreu menos da metade do trajeto original.

Foi desenvolvido também o problema utilizando o Método Simplex, sendo notado grande dificuldade na utilização de vários pontos. No desenvolvimento da temática, conforme o Tabela 4 tem-se uma entrada (Sede) e 3 pontos, lembrando que também sai da Sede e retorna a Sede passando por todos os pontos.

Tabela 4: Problema do caixeiro viajante com 4 pontos (Sede mais 3 pontos) utilizando o Método Simplex.

\begin{tabular}{|c|c|c|c|c|c|c|c|c|c|c|c|c|c|c|c|c|}
\hline Distância Total & 11,71 & & & & & & Caixeir & o Viajante & & & & & & & & \\
\hline \multirow{3}{*}{ Variáveis } & Andaló & Sede & Sede & Sede & P1 & P1 & P1 & P2 & P2 & P2 & P3 & P3 & P3 & & & \\
\hline & Destino & P1 & P2 & P3 & Sede & P2 & P3 & Sede & P1 & P3 & Sede & P1 & P2 & & & \\
\hline & Orig/Des & 0 & 0 & 1 & 0 & 1 & 0 & 1 & 0 & 0 & 0 & 1 & 0 & & & \\
\hline \multirow{2}{*}{ Parâmentros } & Distância & 3,5 & 3,1 & 1,5 & 7,1 & 4,11 & 3,2 & 3,7 & 4,1 & 2,9 & 1,5 & 2,4 & 2,7 & & & \\
\hline & & & & & & & & & & & & & & Total & Sinal & LD \\
\hline \multirow{4}{*}{ Restrições Saida } & Saindo Sede & 1 & 1 & 1 & & & & & & & & & & 1 & $=$ & 1 \\
\hline & Saindo P1 & & & & 1 & 1 & 1 & & & & & & & 1 & $=$ & 1 \\
\hline & Saindo P2 & & & & & & & 1 & 1 & 1 & & & & 1 & $=$ & 1 \\
\hline & Saindo P3 & & & & & & & & & & 1 & 1 & 1 & 1 & $=$ & 1 \\
\hline \multirow{4}{*}{ Restrições Entrada } & Chegando Sede & & & & 1 & & & 1 & & & 1 & & & 1 & $=$ & 1 \\
\hline & Chegando P1 & 1 & & & & & & & 1 & & & 1 & & 1 & $=$ & 1 \\
\hline & Chegando P2 & & 1 & & & 1 & & & & & & & 1 & 1 & $=$ & 1 \\
\hline & Chegando P3 & & & 1 & & & 1 & & & 1 & & & & 1 & $=$ & 1 \\
\hline \multirow{6}{*}{ Restrições Sub Rotas de 2 nós } & Sede,P1 & 1 & & & 1 & & & & & & & & & 0 & ? $<=$ & 1 \\
\hline & Sede,P2 & & 1 & & & & & 1 & & & & & & 1 & $1<=$ & 1 \\
\hline & Sede,P3 & & & 1 & & & & & & & 1 & & & 1 & $<$ & 1 \\
\hline & $\mathrm{P} 1, \mathrm{P} 2$ & & & & & 1 & & & 1 & & & & & 1 & $<=$ & 1 \\
\hline & P1,P3 & & & & & & 1 & & & & & 1 & & 1 & $<=$ & 1 \\
\hline & P2,P3 & & & & & & & & & 1 & & & 1 & 0 & $<=$ & 1 \\
\hline \multirow{4}{*}{ Restricões Sub Rotas de 3 nós } & Sede, $\mathrm{P} 1, \mathrm{P} 2$ & 1 & & & & 1 & & 1 & & & & & & 2 & $?<=$ & 2 \\
\hline & Sede,P1,P3 & 1 & & & & & 1 & & & & 1 & & & 0 & $<=$ & 2 \\
\hline & Sede, P2, P3 & & 1 & & & & & & & 1 & 1 & & & 0 & $<=$ & 2 \\
\hline & $\mathrm{P} 1, \mathrm{P} 2, \mathrm{P3}$ & & & & & 1 & & & & 1 & & 1 & & 2 & ? $<=$ & 2 \\
\hline
\end{tabular}

Fonte: Elaborada pelos Autores (2018) 
No estudo utilizando o Método Simplex com 4 pontos ocorreram 18 restrições a serem feitas: que foram 4 de entrada, 4 de saída, 6 restrições de Sub Rotas de 2 nós e 4 restrições com Sub Rotas de 3 nós, mas se fosse em relação a 7 pontos (6 pontos e 1 entrada) ocorreriam 127 restrições, que são 7 de entrada, 7 de saída, 21 restrições de Sub Rotas de 2 nós, 35 restrições de Sub Rotas 3 nós, 35 restrições de Sub Rotas 4 nós, 21 restrições de Sub Rotas 5 nós e 7 restrições de Sub Rotas 6 nós. A complexidade aumenta consideravelmente. Seguindo no mesmo raciocínio para fazer o problema do caixeiro viajante utilizando o Método Simplex com 11 pontos (10 Pontos e 1 Entrada) teríamos 2364 restrições, ou seja, totalmente inviável a utilização do método Simplex para o cálculo de rotas do Caixeiro Viajante quando se tem uma certa quantidade de pontos, portanto deve-se utilizar o Método Evolutionary para fazer os cálculos quando ocorrerem muitos pontos, pois o mesmo utiliza-se de apenas a quantidade de pontos menos um $(\mathrm{N}-1)$ pontos como restrições, portanto nos exemplos mostrados: com 7 pontos ocorreram 6 restrições, com 11 pontos ocorreram 10 restrições e com 16 pontos ocorreram 15 restrições.

\section{CONCLUSÃO}

Para a realização da logística de entrega entre três pontos de partidas e os vários pontos que são as distribuidoras na cidade de São José do Rio Preto - SP, foi observado que as distribuições seriam feitas de uma forma desordenada, gerando custos desnecessários vindo a afetar significativamente o fluxo de caixa da transportadora, sem a utilização de uma ferramenta eficiente e eficaz para a operação.

Dessa forma, o método do solver que trouxe melhores resultados foi o método evolutionary, trazendo benefícios no que tange as distâncias entre os pontos, além de poder utilizar muitos pontos.

Com a utilização do método simplex, ao contrário do método evolutionary, percebeu-se grande dificuldade ao montar várias rotas, pela sua complexidade de trabalhar com muitos pontos, pois o mesmo gera muitas restrições, inviabilizando assim a resolução do problema.

Portanto, constatou-se que a melhor maneira de calcular a rota com muitos pontos foi com a utilização do Solver e o método evolutionary, pois o mesmo gera poucas restrições facilitando assim na solução do problema. Com o auxílio deste suplemento, foi possível 


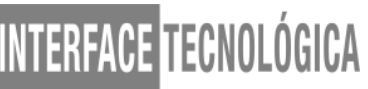

encontrar o caminho mais curto, reduzindo, assim, os gastos com transporte, combustível e tempo.

Conclui-se, então, que para encontrar a melhor solução, ou seja, a melhor rota é essencial que se tenha o auxílio de ferramentas computacionais como o Solver e seus métodos.

\section{REFERÊNCIAS}

ANDRADE, E. L. Introdução à pesquisa operacional: métodos e modelos para análise de decisões. 4ª Edição. Rio de Janeiro: LTC, 2014.

CAIXETA-FILHO, J. V. Pesquisa operacional: técnicas de otimização aplicadas a sistemas agroindustriais. $2^{a}$ Edição. São Paulo: Atlas, 2015.

COLIN, E. C. Pesquisa operacional: 170 aplicações em estratégia, finanças, logística, produção, marketing e vendas. $2^{a}$ Edição. São Paulo: Atlas, 2017.

GIL, A. C. Como elaborar projetos de pesquisa. 4. ed. São Paulo, Atlas, 2002

LACHTERMACHER, G. Pesquisa operacional na tomada de decisões. $4^{\text {a }}$ Edição. São Paulo: Pearson Prentice Hall, 2009.

LANES, R. M.; GRECO, M. Aplicação de um método de otimização topológica evolucionária desenvolvido em script python. 2013. Disponível em: <http://www.seer.ufu.br/index.php/cieng/article/view/22636/20069>. Acesso em: 25 Mai 2018.

MOREIRA, D. A. Pesquisa operacional: curso introdutório. 2a Edição. São Paulo: Cengage Learning, 2015.

XIE, M. Y, e STEVEN, G. P. A Simple evolutionary procedure for structural optimization. Computer \& Structures, v 49, p 885-896, 1993. 